\title{
Quality of Life in Patients with Morphea: A Cross-Sectional Study and a Review of the Current Literature
}

\author{
Justyna Szczęch (D), ${ }^{1}$ Dominik Samotij $\mathbb{D}^{1},{ }^{1}$ Kamila Jaworecka, ${ }^{1}$ Aleksandra Tobiasz, ${ }^{2}$ \\ and Adam Reich $\left.{ }^{1}\right)^{1}$ \\ ${ }^{1}$ Department of Dermatology, University of Rzeszow, Rzeszów, Poland \\ ${ }^{2}$ Students' Scientific Circle of Experimental Dermatology, Department of Dermatology, Venereology and Allergology, \\ Wroclaw Medical University, Wrocław, Poland
}

Correspondence should be addressed to Justyna Szczęch; justyna.m.szczech@gmail.com

Received 29 December 2019; Revised 26 February 2020; Accepted 2 March 2020; Published 13 March 2020

Academic Editor: Emiliano Antiga

Copyright ( 2020 Justyna Szczęch et al. This is an open access article distributed under the Creative Commons Attribution License, which permits unrestricted use, distribution, and reproduction in any medium, provided the original work is properly cited.

\begin{abstract}
Introduction. Morphea (or localized scleroderma) is an inflammatory, immune-mediated disease of unknown etiology. It is characterized by excessive collagen deposition that leads to hardening of the dermis, subcutaneous tissues, or both. Morphea is associated with cosmetic and functional impairment, which might affect the patients' quality of life (QoL). Objective. The aim of the study was to evaluate QoL in patients suffering from morphea. Material and Methods. Sixty-five patients with morphea were recruited into this cross-sectional, prospective parallel study. QoL among adult patients was assessed with the Dermatology Life Quality Index (DLQI) and Euro-QoL-5D questionnaire; patients aged $<17$ years used the Children's Dermatology Life Quality Index (CDLQI). The severity of morphea was assessed using the Localized Scleroderma Cutaneous Assessment Tool. The results of QoL and its association with disease severity were compared between patients with various morphea subtypes. Results. The mean DLQI scoring was $3.8 \pm 4.1$ points and the CDLQI was 2.3 \pm 3.0 . The mean value of Visual Analogue Scale thermometer (EQ VAS) was $66.9 \pm 17.5$ points. The disease activity of morphea based on mLoSSI correlated significantly with QoL impairment according to the DLQI $(R=0.41, p=0.001)$. No significant correlation was observed between morphea-induced damage and QoL $(p=0.99)$. Conclusions. Evaluation of QoL in patients with morphea is still challenging due to lack of good assessment tools dedicated specifically for morphea patients. In general, QoL in morphea patients is significantly correlated with the disease activity, but not with disease-induced skin damage.
\end{abstract}

\section{Introduction}

Morphea (also named as localized scleroderma (LS)) is a rare autoimmune inflammatory disease that essentially affects the skin and/or subcutaneous tissue [1]. It is more common in females, with a female to male ratio of 2-4:1 depending on the studied population [2]. The etiology of morphea still remains unknown; however, the genetic factors, trauma, and vascular abnormalities are considered major trigger factors of this disease [3].

Morphea has a wide spectrum of clinical involvement starting from a relatively mild severity with single plaques. In some cases, the disease leads to facial deformation or joint contractions followed by severe movement impairment. Due to an ample variety of clinical forms of morphea, there are few slightly different classifications proposed in the literature. One of the most current classification was developed in 2017 by the European Dermatology Forum (EDF) [4]. The main subtypes of the disease distinguished in the EDF classification are limited type, generalized type, linear type, deep type, and mixed type [4]. However, the EDF S1-guideline authors also considered eosinophilic fasciitis as another subtype within the spectrum of LS.

Studies assessing the impact of morphea on QoL are giving rather conflicting results. Both the active disease, with typical "lilac ring" around the plaques, and the residual hypo-/hyperpigmented lesions might cause substantial discomfort for the patient. Therefore, patients with morphea might present different impacts of the disease on QoL in different stages of the disease. The main purpose of this study 
was to assess the QoL of the patients with morphea. In this paper, authors have also discussed the results of previously published studies that assessed the QoL of morphea patients. Due to the rarity of this disease, the number of the patients in almost all of the previously conducted studies was relatively small.

\section{Material and Methods}

2.1. Participants. This was a cross-sectional, prospective study conducted in two dermatology clinics in Poland. A total of 65 patients with morphea (6 patients aged below 17 years) were included. The mean age of the patients was $50.9 \pm 20.5$ years (range: $7-82$ years), and $87.7 \%$ were women. Morphea subtype was defined based on the classification proposed in 2017 by the EDF [4]. Patients with lichen sclerosus took part in this study, as this disease is considered a part of a wide spectrum of morphea clinical picture. General patients' characteristics are summarized in Table 1. All patients agreed to take part in the study, and the project was approved by the local ethics committee.

2.2. Assessment of Skin Disease Severity. Several assessment tools were proposed to measure the severity of morphea, although to date, the Localized Scleroderma Cutaneous Assessment Tool (LoSCAT) seems to be the only one with assessed validity and reliability [5]. The LoSCAT allows physicians to measure the LS severity in the active stage and to assess the level of damage developed in the course of the disease. The first part of the LoSCAT, a modified Localized Scleroderma Skin Activity Index (mLoSSI), measures the disease severity in the active, inflammatory stage. Summary scores range from 0 to 162 , with higher scores indicating more disease activity [6]. The second part is a Localized Scleroderma Skin Damage Index (LoSDI), which evaluates three domains of damage that might develop in the course of morphea; again, the summary scores range from 0 to 162 points, with higher scores indicating more severe damage $[5,6]$.

Based on overall LoSCAT scoring, patients were divided into 3 subgroups based on the disease severity: mild, moderate, and severe. The activity corresponded with mLoSSI scores of $0-4,5-12$, and 13 and over; and mild, moderate, and severe damage corresponded with LoSDI scores of $0-10,11-15$, and 16 and over, respectively [7]. This process allowed to compare the QoL specific for different disease severity.

2.3. Assessment of QoL. To evaluate the patients' QoL, we used the DLQI, a self-administered questionnaire designed for adult patients with skin diseases [8]. For children and teenagers, the analogical questionnaire-Children Dermatology Life Quality Index (CDLQI) — was chosen [9]. In both of them, the score ranges from 0 to 30 with higher scores indicating lower QoL.

Euro-QoL-5D is a standardized instrument used to evaluate the health outcome. It consists of 5 questions that evaluate patients' status in terms of mobility, self-care, usual activities, pain/discomfort, and anxiety/depression. The sec-
Table 1: Demographic and clinical characteristics of the patient population.

\begin{tabular}{lc}
\hline & $\begin{array}{c}\text { Morphea, } \\
n(\%)\end{array}$ \\
\hline Gender & $8(12.3 \%)$ \\
Male & $57(87.7 \%)$ \\
Female & $50.9 \pm 20.5$ \\
Age & \\
Clinical subtype of morphea & $31(47.7 \%)$ \\
Plaque type & $15(23.0 \%)$ \\
Generalized type & $3(4.6 \%)$ \\
Linear type & $1(1.5 \%)$ \\
En coup de sabre & $2(3.1 \%)$ \\
Progressive facial hemiatrophy & $7(10.8 \%)$ \\
(Pary-Romberg syndrome) & $1(1.5 \%)$ \\
Atrophoderma of Pasini and Pierini (APP) & $5(7.7 \%)$ \\
Deep type & \\
Lichen sclerosus & $14(21.5 \%)$ \\
Autoimmune comorbidity & $51(78.5 \%)$ \\
Yes & \\
No & $14(21.5 \%)$ \\
Autoimmune diseases in the family & $51(78.5 \%)$ \\
Yes & \\
No & $18(27.7 \%)$ \\
Nosence of antinuclear antibodies & $24(36.9 \%)$ \\
\hline & $23(35.4 \%)$ \\
\hline
\end{tabular}

ond part of EQ-5D contains a Visual Analogue Scale thermometer (EQ VAS) in which a patient subjectively evaluates his/her health from 0 (referred as "the worst health you can imagine") to 100 points ("the best health you can imagine") [10].

\section{Results}

3.1. Disease Onset, Duration, and Clinical Subtypes. The mean disease duration was $3.9 \pm 5.7$ years; the mean age at onset of the disease was $46.0 \pm 21.5$ years. Majority of our patients had plaque-type morphea $(n=31,47.7 \%)$. Disseminated plaque-type morphea was diagnosed in 15 (23.1\%) of all patients, followed by atrophoderma of Pasini and Pierini $(n=7,10.8 \%)$, lichen sclerosus $(n=5,7.7 \%)$, linear morphea $(n=3,4.6 \%)$, progressive facial hemiatrophy $(n=2,3.1 \%)$, deep morphea, and linear morphea en coup de sabre $(n=1$, $1.5 \%)$.

3.2. Disease Severity. The mean mLoSSI was $8.9 \pm 9.6$ points, and the mean LoSDI was $11.5 \pm 10.3$ points. Additionally, patients were divided into 3 subgroups based on the severity of both activity and damage. The characteristics of the morphea severity subgroup based on this classification are shown in Figure 1. Majority of patients had mild or moderate 


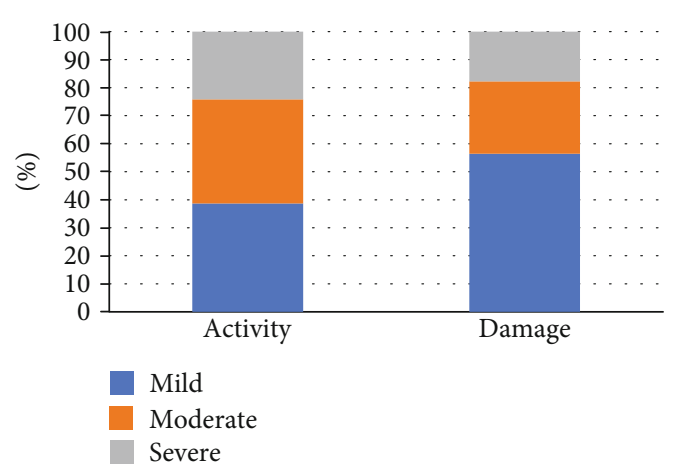

FIgURE 1: Classification of morphea intensity/severity [10].

intensity of both activity and damage based on mLOSSI and LoSDI scoring (activity: mild-38.7\%, moderate-37.1\%, and severe-24.2\%; damage: mild-56.5\%, moderate-25.8\%, and severe-17.7\%).

3.3. Quality of Life Assessment. The mean value of the DLQI among the patients with morphea was $3.7 \pm 4.0$ points. The mean value of the VAS used in EQ-5D was $66.9 \pm 17.5$ points. Regarding the first part of EQ-QoL-5D, majority of patients achieved level 1 , meaning they had no troubles in domains of mobility, self-care, and usual activities. However, in domains connected with discomfort/pain and anxiety/depression, almost half of the patients with morphea achieved level 2 or level 3 as summarized in Table 2.

3.4. QoL and Disease Severity. The disease activity of morphea based on mLoSSI correlated significantly with QoL impairment based on the overall DLQI score $(R=0.41$, $p=0.001)$. There was no such correlation between damage in the course of morphea and DLQI $(p=0.99)$. Moreover, only the disease activity correlated with lower scoring in the VAS of EQ-5D $(R=0.28, p=0.03)$. Figure 2 documents the correlation between different subgroups of disease severity of morphea and DLQI, with significantly higher DLQI scores in patients with severe activity of morphea. The presence of skin lesions on the upper limbs correlated in our patients with greater QoL impairment (higher DLQI score) $(p<0.001)$. We did not observe any significant relationship between the gender, subtype of morphea, disease onset, and duration and QoL impairment. Extracutaneous LS involvement, e.g., joint pain, also did not influence the QoL in our patients.

\section{Discussion}

For this study, a systematic search of the PubMed database was conducted with medical subject headings (MeSH terms) in various combinations: "morphea" or "localized scleroderma" and "quality of life", "DLQI", "dermatology life quality index" or "EQ-5D". All achieved results were checked for relevance of the main topic, type of questionnaires of QoL, and type of scales used for severity of disease assessment. Ultimately, fifteen papers were taken for the final analysis. They were published between 2008 and 2019 , and the number of patients ranged from 27 up to
TABLE 2: Level achieved in each domain of EQ-QoL-5D by patients with morphea.

\begin{tabular}{lccccc}
\hline & Mobility & Self-care & $\begin{array}{c}\text { Usual } \\
\text { activities }\end{array}$ & $\begin{array}{c}\text { Pain/ } \\
\text { discomfort }\end{array}$ & $\begin{array}{c}\text { Anxiety/ } \\
\text { depression }\end{array}$ \\
\hline Level 1 & $90 \%$ & $95 \%$ & $82 \%$ & $52 \%$ & $53 \%$ \\
Level 2 & $7 \%$ & $3 \%$ & $15 \%$ & $\mathbf{3 5 \%}$ & $\mathbf{3 3 \%}$ \\
Level 3 & $3 \%$ & $2 \%$ & $3 \%$ & $\mathbf{1 0 \%}$ & $\mathbf{1 0 \%}$ \\
Level 4 & $0 \%$ & $0 \%$ & $0 \%$ & $2 \%$ & $3 \%$ \\
Level 5 & $0 \%$ & $0 \%$ & $0 \%$ & $2 \%$ & $0 \%$ \\
\hline
\end{tabular}

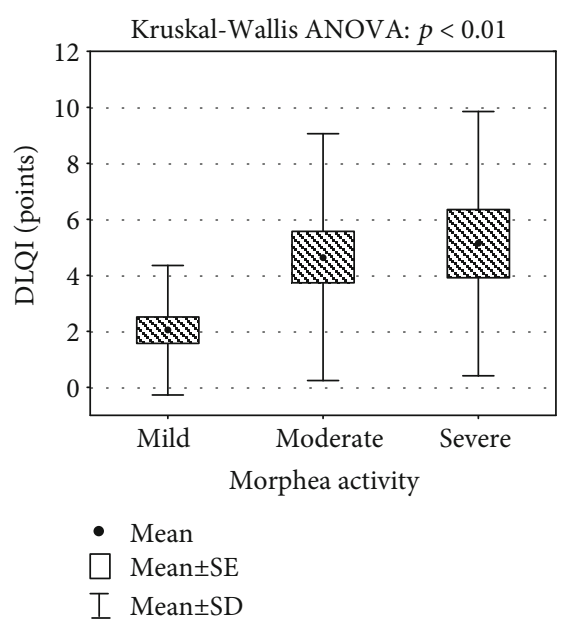

FIgURE 2: Morphea activity and scoring of the Dermatology Life Quality Index (DLQI).

581. Some of the studies used the Morphea in Adults and Children (MAC) cohort prepared by the University of Texas Southwestern Medical Center.

In the last 10 years, only 15 papers focusing on QoL among the patients with morphea were published. All of the studies have some limitations which are shortly summarized in Table 3 [11-25]. The major limitation of almost all of the previous studies is the relatively small groups of patients taken into the final analysis. It implicates rather poor diversity of morphea subtypes, with the highest prevalence of plaque-type morphea in the majority of studies. Only in 3 studies that the comparison of QoL between morphea patients and the control group, of either healthy individuals or patients with another dermatological disorders, is available [12, 17, 21]. In two of the studies, one performed on a pediatric population; no data regarding the disease severity assessment performed by a qualified physician was obtained $[12,15]$.

Except the abovementioned limitations, these studies give a further perspective on QoL among the patients with morphea, in which majority of them showed that morphea exerts a mild to moderate impact on patients' QoL. Modest differences in QoL were observed between the subtypes of morphea. As presented in the study by Das et al. and Bali et al., both disseminated morphea and linear subtype had higher, albeit not significantly, total scoring of the DLQI $[18,24]$. 


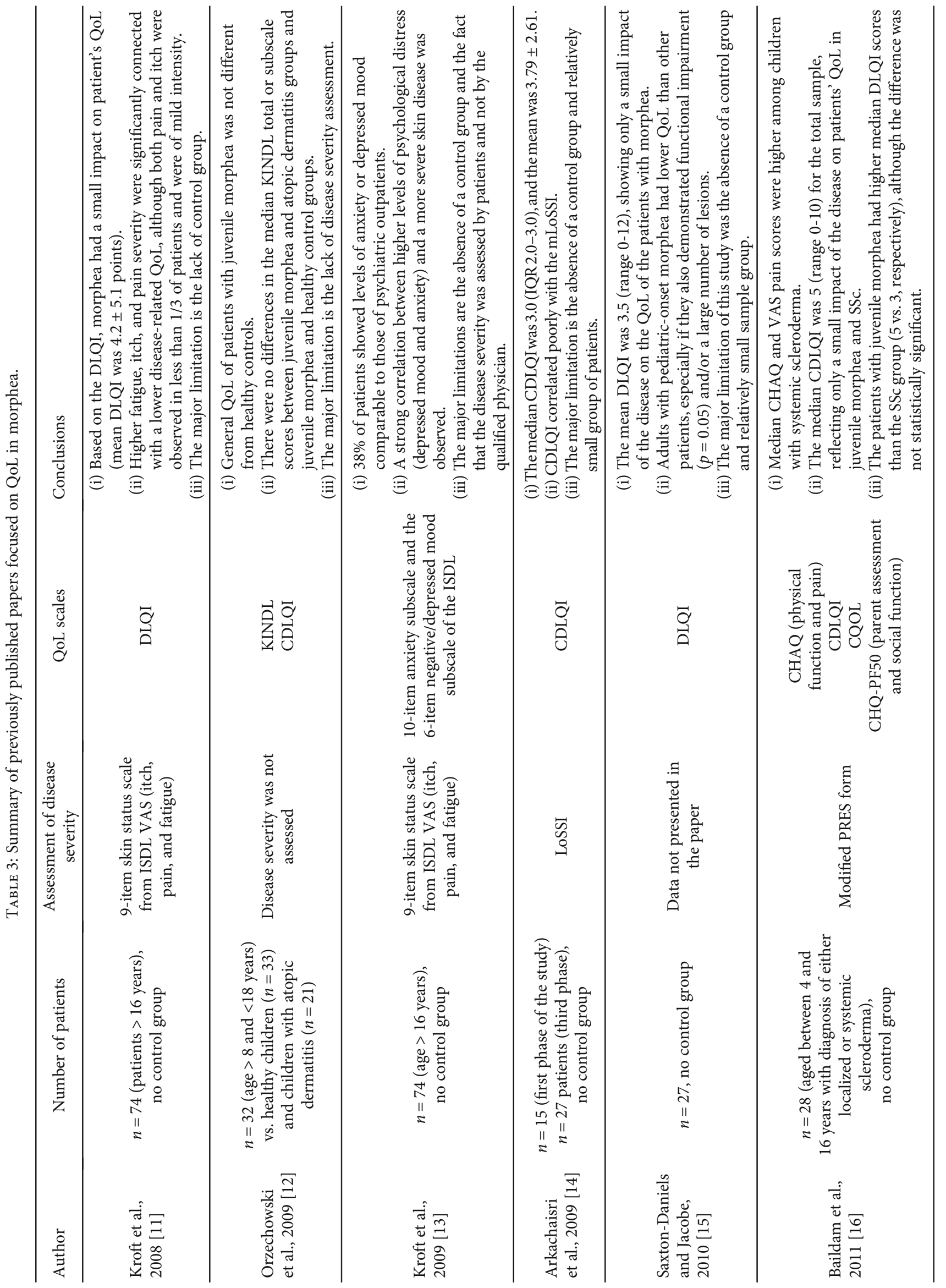




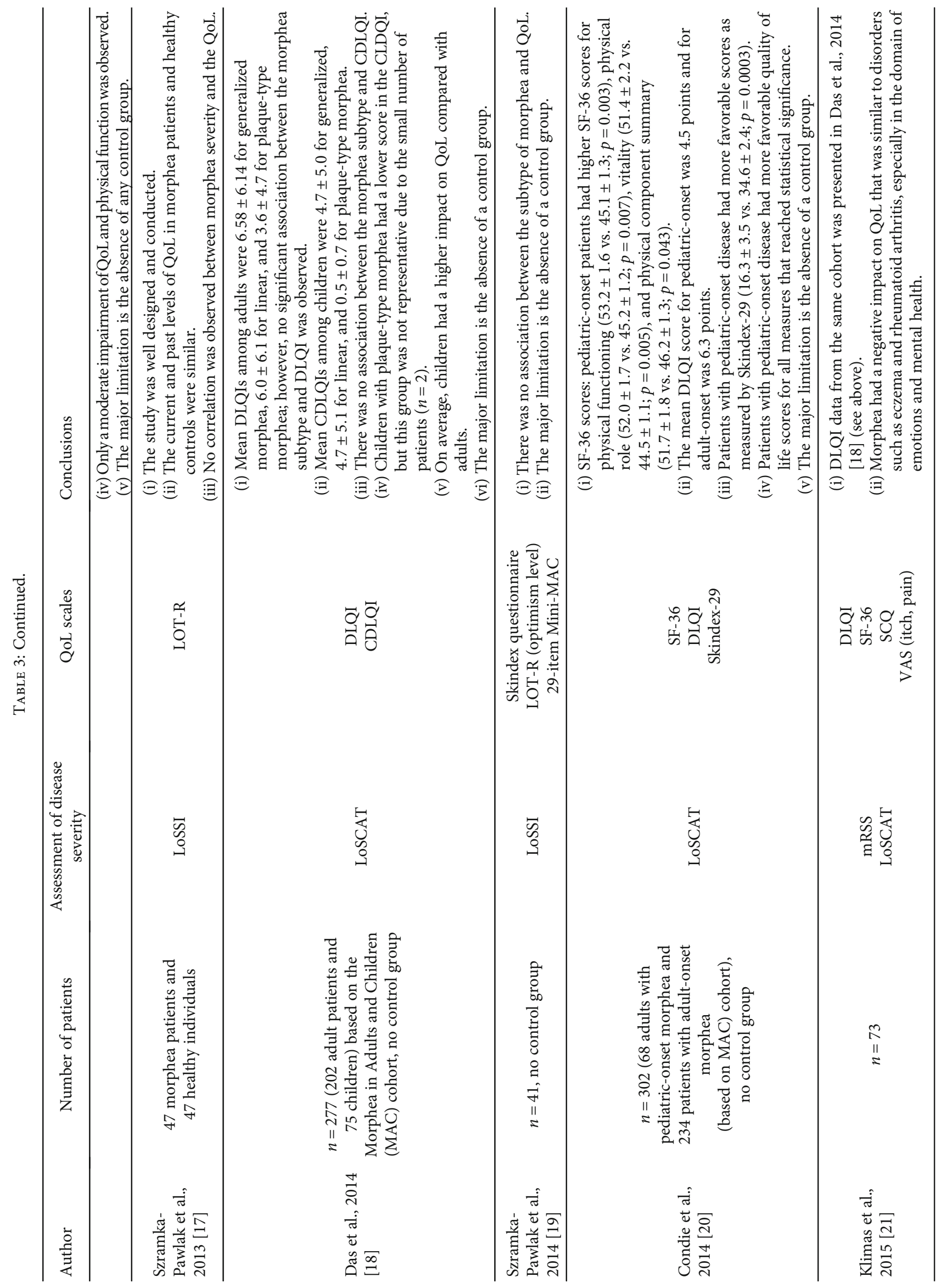




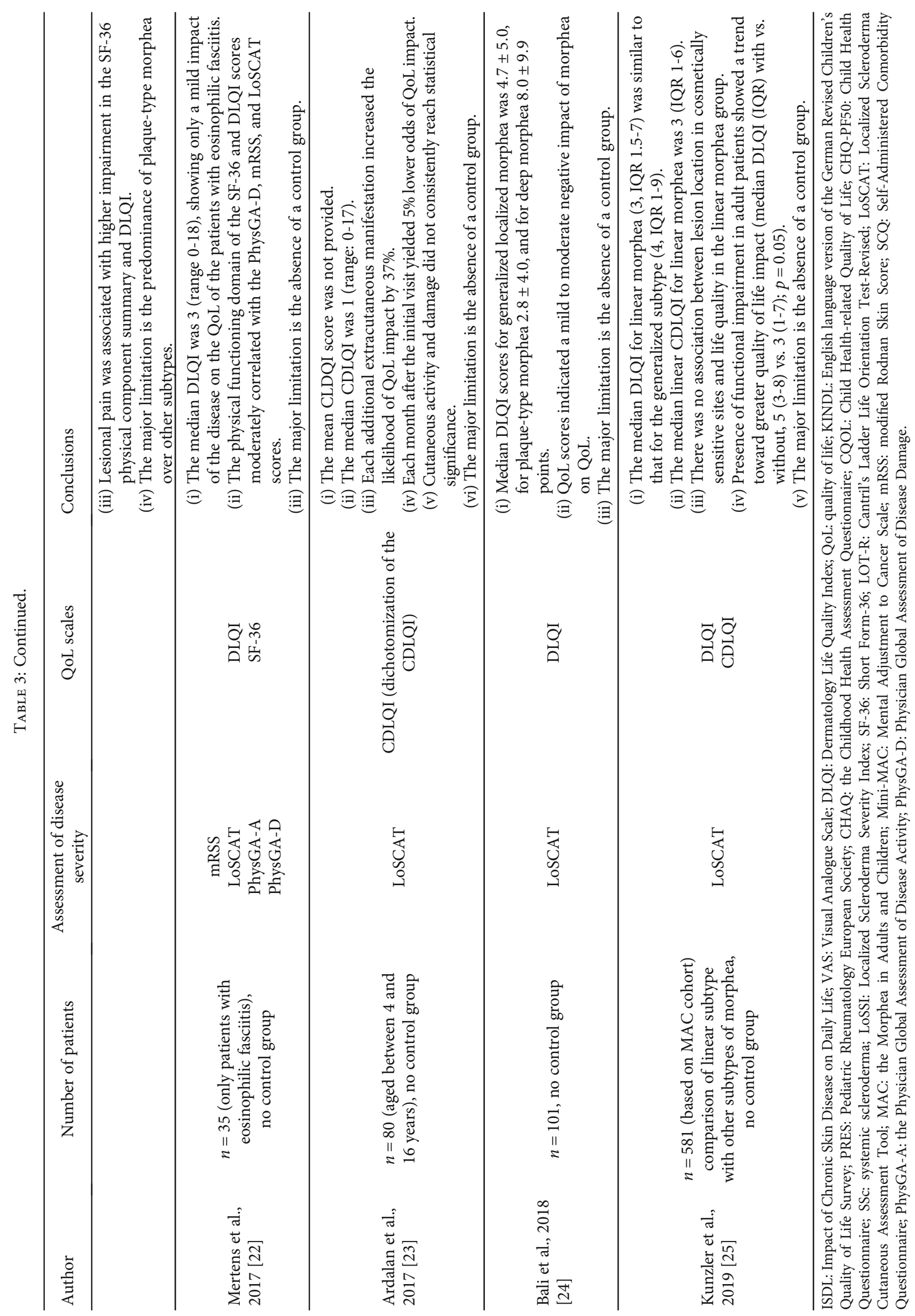


The relatively small impact on QoL among the patients with morphea may indicate the need of a specific tool to assess specifically problems related to this dermatologic condition. Previously published papers that evaluated the influence of morphea on QoL gave undetermined answer, whether the subtype of the disease or its severity might have an impact on patients' QoL. In our study, we have conducted a new approach to classify morphea severity based on differentiation between mild, moderate, and severe diseases proposed by Teske and Jacobe [7]. Possibly, this division allowed us to show that severe morphea, in terms of activity but not damage, ultimately had an impact on patients' QoL. Similar results were obtained by Mertens et al. [22]. Previously, Das et al. observed that increased LoSSI and LoSDI scores correlated with a greater impact on QoL among the adult patients; however, he did not observe similar correlation between physician-based measures and CDLQI [18]. These results are similar to the one obtained in the study by Klimas et al. in an adult population, who additionally pointed out that physical disability, e.g., joint constrictions observed especially in the linear subtype of morphea, did have a negative impact on QoL [21]. Interestingly, significant differences between patients with pediatric-onset and adult-onset morphea were noticed in the study conducted by Condie et al. [20]. Based on three QoL assessment tools: SF-36, DLQI, and Skindex-29, the authors observed that QoL among the patients with adult-onset morphea was poorer comparing to that among patients with pediatric-onset disease [20].

In comparison with other dermatological conditions, pediatric patients with morphea have higher median DLQI scores than children with, e.g., systemic scleroderma [16]. However, as shown by Condie et al. patients with adultonset morphea had SF-36 component summary scores similar to those seen in other dermatologic conditions, including psoriasis or atopic dermatitis [20].

Some of the studies focused also on additional symptoms of skin involvement in the course of morphea. Both Kroft et al. and Das et al. noted that the presence of fatigue, pain, and itch in the course of morphea significantly correlates with the QoL impairment assessed with the DLQI $[13,18]$.

In July of 2019, the results of the long-term, prospective, single-site MAC cohort study performed by Kunzler et al. was published [25]. This longitudinal analysis showed results from a 3-year follow-up, however, in the final assessment of either DLQI or CDLQI which took part only $38.7 \%$ of initial participants [25]. Authors received similar results to those published earlier that used MAC cohort for the analysis of QoL in patients with morphea; they observed only a mild effect of disease on patients' QoL. Although they underlined that functional impairment observed mostly among patients with the linear subtype of morphea might have important impact on QoL, that is not assessed by the DLQI or CDLQI.

The major limitations of our study are the relatively small group of patients with poor subtype diversity, which is a similar limitation as in previous studies. It is a result of a rarity of morphea and usual overall predominance of plaque-type disease [18].

The QoL in patients with morphea due to the lack of a good assessment tool dedicated specifically for morphea patients is hard to evaluate. As shown based on our results and in previous studies, the relatively small impact of QoL among the patients with morphea may indicate the need of a specific tool to assess this parameter specifically addressed to this dermatologic condition. This need might be finally fulfilled as new QoL measurement tools are in development. In 2019, Zigler et al. published a paper focusing on the development of a new health-related quality of life measure for individuals with pediatric morphea, which hopefully will contribute to a better therapeutic approach based on patient' expectations [26].

In conclusion, the evaluation of QoL in patients with morphea is still challenging due to the lack of good assessment tools dedicated specifically for morphea patients. In general, QoL in morphea patients is significantly correlated with the disease activity, but not with disease-induced skin damage. Further studies are needed to analyze QoL in morphea patients, especially regarding its rare clinical subtypes.

\section{Data Availability}

The datasets generated during and/or analyzed during the current study are available from the corresponding author on reasonable request (email: justyna.m.szczech@gmail.com).

\section{Conflicts of Interest}

The authors declare that they have no conflicts of interest.

\section{Acknowledgments}

The study was conducted using the grant for Young Researchers sponsored by the University of Rzeszow. The grant number is $500-3-08-084$.

\section{References}

[1] D. L. Tuffanelli, "Localized scleroderma," Seminars in Cutaneous Medicine and Surgery, vol. 17, no. 1, pp. 27-33, 1998.

[2] N. Fett and V. P. Werth, "Update on morphea: part I. Epidemiology, clinical presentation, and pathogenesis," Journal of the American Academy of Dermatology, vol. 64, no. 2, pp. 217228, 2011.

[3] M. F. Careta and R. Romiti, "Localized scleroderma: clinical spectrum and therapeutic update," Anais Brasileiros de Dermatologia, vol. 90, no. 1, pp. 62-73, 2015.

[4] R. Knobler, P. Moinzadeh, N. Hunzelmann et al., "European Dermatology Forum S1-guideline on the diagnosis and treatment of sclerosing diseases of the skin, part 1: localized scleroderma, systemic sclerosis and overlap syndromes," Journal of the European Academy of Dermatology and Venereology, vol. 31, no. 9, pp. 1401-1424, 2017.

[5] E. Herédi, F. Rencz, O. Balogh et al., "Exploring the relationship between EQ-5D, DLQI and PASI, and mapping EQ-5D utilities: a cross-sectional study in psoriasis from Hungary," The European Journal of Health Economics, vol. 15, Supplement 1, pp. 111-119, 2014.

[6] T. Arkachaisri, S. Vilaiyuk, K. S. Torok, and T. A. Medsger Jr., "Development and initial validation of the localized scleroderma skin damage index and physician global assessment of 
disease damage: a proof-of-concept study," Rheumatology (Oxford, England), vol. 49, no. 2, pp. 373-381, 2010.

[7] N. M. Teske and H. T. Jacobe, "Using the Localized Scleroderma Cutaneous Assessment Tool (LoSCAT) to classify morphea by severity and identify clinically significant change," The British Journal of Dermatology, vol. 3, 2019.

[8] M. K. A. Basra, R. Fenech, R. M. Gatt, M. S. Salek, and A. Y. Finlay, "The Dermatology Life Quality Index 1994-2007: a comprehensive review of validation data and clinical results," British Journal of Dermatology, vol. 159, no. 5, pp. 997-1035, 2008.

[9] M. S. Lewis-Jones and A. Y. Finlay, “The Children's Dermatology Life Quality Index (CDLQI): initial validation and practical use," The British Journal of Dermatology, vol. 132, no. 6, pp. 942-949, 1995.

[10] R. Rabin and F. de Charro, "EQ-SD: A measure of health status from the EuroQol Group," Annals of Medicine, vol. 33, no. 5, pp. 337-343, 2001.

[11] E. B. Kroft, E. M. de Jong, and A. W. Evers, "Physical burden of symptoms in patients with localized scleroderma and eosinophilic fasciitis," Archives of Dermatology, vol. 144, no. 10, pp. 1394-1395, 2008.

[12] N. M. Orzechowski, D. M. Davis, T. G. Mason, C. S. Crowson, and A. M. Reed, "Health-related quality of life in children and adolescents with juvenile localized scleroderma," Rheumatology, vol. 48, no. 6, pp. 670-672, 2009.

[13] E. B. M. Kroft, E. M. G. J. de Jong, and A. W. M. Evers, "Psychological distress in patients with morphea and eosinophilic fasciitis," Archives of Dermatology, vol. 145, no. 9, pp. 10171022, 2009.

[14] T. Arkachaisri, S. Vilaiyuk, S. Li et al., "The localized scleroderma skin severity index and physician global assessment of disease activity: a work in progress toward development of localized scleroderma outcome measures," The Journal of Rheumatology, vol. 36, no. 12, pp. 2819-2829, 2009.

[15] S. Saxton-Daniels and H. T. Jacobe, "An evaluation of longterm outcomes in adults with pediatric-onset morphea," Archives of Dermatology, vol. 146, no. 9, pp. 1044-1045, 2010.

[16] E. M. Baildam, H. Ennis, H. E. Foster et al., "Influence of childhood scleroderma on physical function and quality of life," The Journal of Rheumatology, vol. 38, no. 1, pp. 167173, 2011.

[17] B. Szramka-Pawlak, A. Dańczak-Pazdrowska, T. Rzepa, A. Szewczyk, A. Sadowska-Przytocka, and R. Żaba, "Healthrelated quality of life, optimism, and coping strategies in persons suffering from localized scleroderma," Psychology, Health \& Medicine, vol. 18, no. 6, pp. 654-663, 2013.

[18] S. Das, I. Bernstein, and H. Jacobe, "Correlates of self-reported quality of life in adults and children with morphea," Journal of the American Academy of Dermatology, vol. 70, no. 5, pp. 904910, 2014.

[19] B. Szramka-Pawlak, A. Dańczak-Pazdrowska, T. Rzepa, A. Szewczyk, A. Sadowska-Przytocka, and R. Zaba, "Quality of life and optimism in patients with morphea," Applied Research in Quality of Life, vol. 9, no. 4, pp. 863-870, 2014.

[20] D. Condie, D. Grabell, and H. Jacobe, "Comparison of outcomes in adults with pediatric-onset morphea and those with adult-onset morphea: a cross-sectional study from the morphea in adults and children cohort," Arthritis \& Rhematology, vol. 66 , no. 12, pp. 3496-3504, 2014.
[21] N. K. Klimas, A. D. Shedd, I. H. Bernstein, and H. Jacobe, "Health-related quality of life in morphoea," The British Journal of Dermatology, vol. 172, no. 5, pp. 1329-1337, 2015.

[22] J. S. Mertens, R. M. Thurlings, W. Kievit, M. M. B. Seyger, T. R. D. Radstake, and E. M. G. J. de Jong, "Long-term outcome of eosinophilic fasciitis: a cross-sectional evaluation of 35 patients," Journal of the American Academy of Dermatology, vol. 77, no. 3, pp. 512-517.e5, 2017.

[23] K. Ardalan, C. K. Zigler, and K. S. Torok, "Predictors of longitudinal quality of life in juvenile localized scleroderma," Arthritis Care \& Research, vol. 69, no. 7, pp. 1082-1087, 2017.

[24] G. Bali, S. Kárpáti, M. Sárdy, V. Brodszky, B. Hidvégi, and F. Rencz, "Association between quality of life and clinical characteristics in patients with morphea," Quality of Life Research, vol. 27, no. 10, pp. 2525-2532, 2018.

[25] E. Kunzler, S. Florez-Pollack, N. Teske, J. O'Brien, S. Prasad, and H. Jacobe, "Linear morphea: clinical characteristics, disease course, and treatment of the Morphea in Adults and Children cohort," Journal of the American Academy of Dermatology, vol. 80, no. 6, pp. 1664-1670.e1, 2019.

[26] C. K. Zigler, K. Ardalan, S. Lane, K. L. Schollaert, and K. S. Torok, "A novel patient-reported outcome for paediatric localized scleroderma: a qualitative assessment of content validity," The British Journal of Dermatology, vol. 182, no. 3, pp. 625635, 2019. 